\title{
REACHABILITY MATRICES AND CYCLIC MATRICES*
}

\author{
AUGUSTO FERRANTE ${ }^{\dagger}$ AND HARALD K. WIMMER ${ }^{\ddagger}$
}

\begin{abstract}
We study reachability matrices $R(A, b)=\left[b, A b, \ldots, A^{n-1} b\right]$, where $A$ is an $n \times n$ matrix over a field $K$ and $b$ is in $K^{n}$. We characterize those matrices that are reachability matrices for some pair $(A, b)$. In the case of a cyclic matrix $A$ and an $n$-vector of indeterminates $x$, we derive a factorization of the polynomial $\operatorname{det}(R(A, x))$.
\end{abstract}

Key words. Reachability matrix, Krylow matrix, cyclic matrix, nonderogatory matrix, companion matrix, Vandermonde matrix, Hautus test.

AMS subject classifications. 15A03, 15A15, 93B05.

1. Introduction. Let $K$ be a field, and $A \in K^{n \times n}, b \in K^{n}$. The matrix

$$
R(A, b)=\left[b, A b, \ldots, A^{n-1} b\right] \in K^{n \times n}
$$

is the reachability matrix of the pair $(A, b)$. A matrix $A$ is called cyclic (e.g. in [3], [4]) or nonderogatory (e.g. in [2], [9]), if there exists a vector $b \in K^{n}$ such that

$$
\operatorname{span}\left\{b, A b, A^{2} b, \ldots, A^{n-1} b\right\}=K^{n}
$$

In that case the pair $(A, b)$ is said to be reachable. Let

$$
a(z)=z^{n}-\left(a_{n-1} z^{n-1}+\cdots+a_{1} z+a_{0}\right)
$$

be the characteristic polynomial of $A$. The matrix

$$
F_{a}=\left[\begin{array}{cccccc}
0 & \cdot & & & & a_{0} \\
1 & 0 & . & & & a_{1} \\
& \cdot & \cdot & \cdot & & \\
& & \cdot & \cdot & \cdot & \\
& & & \cdot & \cdot & \cdot \\
0 & 0 & & & 1 & a_{n-1}
\end{array}\right]
$$

\footnotetext{
${ }^{*}$ Received by the editors September 23, 2009. Accepted for publication February 11, 2010. Handling Editor: Daniel Szyld.

${ }^{\dagger}$ Dipartimento di Ingegneria dell'Informazione, Università di Padova, I-35131 Padova, Italy (augusto@dei.unipd.it).

${ }^{\ddagger}$ Mathematisches Institut, Universität Würzburg, D-97074 Würzburg, Germany (wimmer@mathematik.uni-wuerzburg.de).
} 
is the companion matrix of the second type [1] associated with (1.2). It is well known (see e.g. [4, p. 299]) that $A$ is cyclic if and only if $A$ is similar to the companion matrix $F_{a}$. Or equivalently, if $x_{0}, \ldots, x_{n-1}$ are indeterminates over $K$ and $x:=$ $\left[x_{0}, \ldots, x_{n-1}\right]^{\top}$, then $A$ is cyclic if and only if the polynomial $\operatorname{det} R(A, x)$ is not the zero polynomial.

In this note we are concerned with the following questions. When is a given matrix $M \in K^{n \times n}$ a reachability matrix? How can one factorize the polynomial $\operatorname{det} R(A, x)$ ?

2. Companion and reachability matrices. In this section we characterize those matrices that are reachability matrices for some pair $(A, b)$. We first show that each nonsingular matrix $M$ is a reachability matrix. Let

$$
e_{0}=[1,0, \ldots, 0]^{\top}, \ldots, e_{n-1}=[0, \ldots, 0,1]^{\top},
$$

be the unit vectors of $K^{n}$.

Theorem 2.1. Let $M=\left[v_{0}, v_{1}, \ldots, v_{n-1}\right] \in K^{n \times n}$ be nonsingular. Then $M=$ $R(A, b)$ if and only if $b=v_{0}$ and $A=M F_{c} M^{-1}$ for some nonsingular companion matrix $F_{c}$. In particular, $M=R\left(A, v_{0}\right)$ with

$$
A=\left[v_{1}, \ldots, v_{n-1}, v_{0}\right] M^{-1} .
$$

Proof. We have $e_{0}=M^{-1} v_{0}$. Hence, if $b=v_{0}$ and $A=M F_{c} M^{-1}$ then $A^{i} b=M F_{c}^{i} e_{0}=M e_{i}=v_{i}$, and thus $M=R(A, b)$. We obtain (2.1) if we choose $F_{c}=\left(e_{1}, e_{2}, \ldots, e_{n-1}, e_{0}\right)$. Conversely, if $M=R(A, b)$, then $b=v_{0}$, and $A M=M F_{a}$ for some companion matrix $F_{a}$. Hence if $M$ is nonsingular then $A=M F_{a} M^{-1}$.

Theorem 2.2. Let $M=\left[v_{0}, v_{1}, \ldots, v_{n-1}\right] \in K^{n \times n}$ and $\operatorname{rank} M=r$. The following statements are equivalent.

(i) $M$ is a reachability matrix.

(ii) Either $\operatorname{rank} M=n$, i.e. the matrix $M$ is nonsingular, or

$$
\operatorname{rank} M=\operatorname{rank}\left[v_{0}, v_{1}, \ldots, v_{r-1}\right]=r<n
$$

and

$$
\begin{aligned}
& \operatorname{Ker}\left[v_{k-1}, v_{k}, \ldots, v_{k-1+r}\right] \subseteq \operatorname{Ker}\left[v_{k}, v_{k+1}, \ldots, v_{k+r}\right], \\
& k=1,2, \ldots, n-1-r .
\end{aligned}
$$


Proof. If (2.2) holds then (2.3) means that there exist $c_{i} \in K, i=0, \ldots, r-1$, such that

$$
v_{r+k}=\sum_{i=0}^{r-1} c_{i} v_{i+k}, k=0,1 \ldots, n-r-1 .
$$

(i) $\Rightarrow$ (ii) If $M$ is a reachability matrix and $\operatorname{rank} M=r<n$ then it is obvious that the conditions (2.2) and (2.4) are satisfied.

(ii) $\Rightarrow$ (i) If $\operatorname{rank} M=n$ then it follows from Theorem 2.1 that $M$ is a reachability matrix. Now assume (2.2) and (2.4). Let $Q \in K^{n \times n}$ be nonsingular such that

$$
Q\left[v_{0}, \ldots, v_{r-1}\right]=\left[\begin{array}{c}
I_{r} \\
0
\end{array}\right] .
$$

Let $\hat{e}_{0}, \ldots, \hat{e}_{r-1}$ be the canonical unit vectors of $K^{r}$. Then (2.4) implies

$$
\begin{aligned}
Q M=\left[\begin{array}{cccccc}
w_{0} & \ldots & w_{r-1} & w_{r} & \ldots & w_{n-1} \\
0 & \ldots & 0 & 0 & \ldots & 0
\end{array}\right]= \\
{\left[\begin{array}{ccccccc}
\hat{e}_{0} & \ldots & \hat{e}_{r-1} & w_{r} & \ldots & w_{n-1} \\
0 & \ldots & 0 & 0 & \ldots & 0
\end{array}\right], }
\end{aligned}
$$

and the vectors $w_{i}$ satisfy

$$
w_{r+k}=\sum_{i=0}^{r-1} c_{i} w_{i+k}, \quad k=0,1 \ldots, n-r-1 .
$$

Set

$$
\hat{A}=\left[\begin{array}{ccccc}
0 & 0 & & & c_{0} \\
1 & 0 & & & c_{1} \\
& \cdot & \cdot & & \\
& & \cdot & \cdot & \\
0 & 0 & & 1 & c_{r-1}
\end{array}\right] .
$$

Then

$$
w_{r}=\left[c_{0}, c_{1}, \ldots, c_{r-1}\right]^{\top}=\hat{A} \hat{e}_{r-1}=\hat{A} \hat{A}^{r-1} \hat{e}_{0}=\hat{A}^{r} \hat{e}_{0}
$$

and

$$
\left[\hat{e}_{0}, \hat{A} \hat{e}_{0}, \ldots, \hat{A}^{r-1} \hat{e}_{0}, \hat{A}^{r} \hat{e}_{0}, \ldots, \hat{A}^{n-1} \hat{e}_{0}\right]=\left[\hat{e}_{0}, \ldots, \hat{e}_{r-1}, w_{r}, \ldots, w_{n-1}\right] .
$$

Hence the matrix $Q M$ in (2.5) can be written as

$$
Q M=R(A, b) \quad \text { with } \quad A=\left[\begin{array}{cc}
\hat{A} & 0 \\
0 & 0
\end{array}\right] \in K^{n \times n}, b=\left[\begin{array}{c}
\hat{e}_{0} \\
0
\end{array}\right] \in K^{n},
$$

and we obtain $M=R\left(Q^{-1} A Q, Q^{-1} b\right)$. $\square$ 
3. A factorization theorem. We first characterize companion matrices in terms of reachability matrices. Let $b=\left(b_{0}, b_{1}, \ldots, b_{n-1}\right)^{\top} \in K^{n}$. We call

$$
b(z)=\left(1, z, \ldots, z^{n-1}\right) b=b_{0}+b_{1} z+\cdots+b_{n-1} z^{n-1}
$$

the polynomial associated to $b$.

Proposition 3.1. Let $a(z)=z^{n}-\sum a_{i} z^{i}$ be the characteristic polynomial of $A \in K^{n \times n}$, and let $F_{a}$ be the companion matrix in (1.3). Then $A=F_{a}$ if and only if

$$
R(A, b)=b(A) \quad \text { for all } \quad b \in K^{n} .
$$

Proof. It is obvious that $A$ is a companion matrix of the form (1.3) if and only if

$$
A\left[e_{0}, e_{1}, \ldots, e_{n-2}\right]=\left[e_{1}, e_{2}, \ldots, e_{n-1}\right] .
$$

Assume now that (3.2) is satisfied. Choose $b=e_{0}$. Then $b(z)=1$ and $b(A)=I$. Therefore

$$
R\left(A, e_{0}\right)=\left[e_{0}, A e_{0}, \ldots, A^{n-1} e_{0}\right]=I=\left[e_{0}, e_{1}, \ldots, e_{n-1}\right] .
$$

Hence we obtain (3.3), and we conclude that $A=F_{a}$. To prove the converse we have to show that

$$
R\left(F_{a}, b\right)=b\left(F_{a}\right)
$$

holds for all $b=\sum_{i=0}^{n-1} b_{i} e_{i}$. We have $e_{i}=F_{a}^{i} e_{0}, i=0, \ldots, n-1$. From $R\left(F_{a}, e_{0}\right)=I$ and $R\left(F_{a}, e_{i}\right)=F_{a}^{i} R\left(F_{a}, e_{0}\right)$ follows $R\left(F_{a}, e_{i}\right)=F_{a}^{i}$. Therefore

$$
R\left(F_{a}, b\right)=\sum_{i=0}^{n-1} b_{i} R\left(F_{a}, e_{i}\right)=\sum_{i=0}^{n-1} b_{i} F_{a}^{i}=b\left(F_{a}\right) . \square
$$

Suppose $A$ is cyclic. Let $S$ be nonsingular such that $S A S^{-1}=F_{a}$, and let the polynomial $(S b)(z)$ be defined in analogy to (3.1). Then $S R(A, b)=R\left(F_{a}, S b\right)$. From (3.2) we obtain

$$
R(A, b)=S^{-1}(S b)\left(F_{a}\right) .
$$

Note that for all $b \in K^{n}$ we have $A R(A, b)=R(A, b) F_{a}$. Hence, if the pair $(A, b)$ is reachable then the matrix $S=R(A, b)^{-1}$ satisfies

$$
S A S^{-1}=F_{a} .
$$

The identity (3.6) can be found in [6, Section 6.1]. 
For the following observation we are indebted to a referee. Suppose $A$ is a matrix with distinct eigenvalues, and $X^{-1} A X=D$ is a Jordan form. Then the corresponding companion matrix $F_{a}$ is similar to the diagonal matrix $D$. The similarity transformation $V F_{a} V^{-1}=D$ is accomplished by a Vandermonde matrix $V$ whose nodes are the eigenvalues of $A$ (see e.g. [12, Section 1.11]). One can write $V$ as a reachability matrix, that is, $V=R(D, e)$, where $e=(1,1, \ldots, 1)^{\top}$.

Let $a_{j}(z), j=1, \ldots, r$, be the monic irreducible factors of the polynomial $a(z)$ in (1.2). Suppose $\operatorname{deg} a_{j}(z)=\ell_{j}$ and

$$
a(z)=a_{1}(z)^{m_{1}} \cdots a_{r}(z)^{m_{r}}
$$

such that $\sum_{j=1}^{r} m_{j} \ell_{j}=n$. Let $F_{a_{j}} \in K^{\ell_{j} \times \ell_{j}}$ be the corresponding companion matrices. The main result of this section is the following.

Theorem 3.2. Let $A$ be cyclic with characteristic polynomial a(z), and let (3.7) be the prime factorization of a $(z)$. Suppose

$$
S A S^{-1}=F_{a} \quad \text { and } \quad \operatorname{det} S=1 .
$$

Set $g_{j}(x)=\operatorname{det}(S x)\left(F_{a_{j}}\right), j=1, \ldots, r$. Then

$$
\operatorname{det} R(A, x)=\left(g_{1}(x)\right)^{m_{1}} \cdots\left(g_{r}(x)\right)^{m_{r}} .
$$

The polynomials $g_{1}(x), \ldots, g_{r}(x)$ are irreducible, and homogeneous of degree $\ell_{1}, \ldots, \ell_{r}$, respectively.

Proof. Define

$$
C\left(a_{j}, m_{j}\right)=\left(\begin{array}{ccccc}
F_{a_{j}} & I_{\ell_{j}} & 0 & . & 0 \\
0 & F_{a_{j}} & I_{\ell_{j}} & . & 0 \\
\cdot & \cdot & \cdot & . & . \\
\cdot & \cdot & \cdot & F_{a_{j}} & I_{\ell_{j}} \\
\cdot & \cdot & \cdot & \cdot & F_{a_{j}}
\end{array}\right)_{m_{j} \ell_{j} \times m_{j} \ell_{j}} .
$$

Then

$$
T F_{a} T^{-1}=\operatorname{diag}\left(C\left(a_{1}, m_{1}\right), \ldots, C\left(a_{r}, m_{r}\right)\right)
$$

for some $T \in K^{n \times n}$. The right hand side of (3.10) is the rational canonical form (the Frobenius canonical form) of $F_{a}$. Let $\hat{b} \in K^{n}$. From (3.4) be obtain

$$
R\left(F_{a}, \hat{b}\right)=\hat{b}\left(F_{a}\right)=T^{-1} \hat{b}\left[\operatorname{diag}\left(C\left(a_{1}, m_{1}\right), \ldots, C\left(a_{r}, m_{r}\right)\right)\right] T .
$$

Hence

$$
\begin{aligned}
& \operatorname{det} R\left(F_{a}, \hat{b}\right)=\operatorname{det} \hat{b}\left(C\left(a_{1}, m_{1}\right)\right) \cdots \operatorname{det} \hat{b}\left(C\left(a_{r}, m_{r}\right)\right)= \\
&\left(\operatorname{det} \hat{b}\left(F_{a_{1}}\right)\right)^{m_{1}} \cdots\left(\operatorname{det} \hat{b}\left(F_{a_{r}}\right)\right)^{m_{r}} .
\end{aligned}
$$


Suppose $S A S^{-1}=F_{a}$ and $\operatorname{det} S=1$. Then (3.5) implies $\operatorname{det} R(A, x)=\operatorname{det} R\left(F_{a}, S x\right)$. Taking $\hat{b}=S x$ in (3.11) we obtain (3.9).

Suppose one of the polynomials $g_{j}(x)$ is reducible. E.g. let $g_{1}(x)=p(x) q(x)$ and $\operatorname{deg} p \geq 1, \operatorname{deg} q \geq 1$. Then $x=S^{-1}(-1, z, 0, \ldots, 0)^{\top}$ yields $(S x)\left(F_{a_{1}}\right)=-F_{a_{1}}+z I$. Hence $g_{1}(x)=\operatorname{det}\left(-F_{a_{1}}+z I\right)=a_{1}(z)$. On the other hand $g_{1}(x)=\tilde{p}(z) \tilde{q}(z)$, and $\operatorname{deg} \tilde{p} \geq 1, \operatorname{deg} \tilde{q} \geq 1$. This is a contradiction to the irreducibility of $a_{1}(z)$.

Since $F_{a_{j}}$ is of size $\ell_{j} \times \ell_{j}$ we obtain $g_{j}(\lambda x)=\lambda^{\ell_{j}} g_{j}(x)$. Thus, $g_{j}(x)$ is homogeneous of degree $\ell_{j}$. $\square$

We now assume that the characteristic polynomial $a(z)$ of $A$ splits over $K$. If $\lambda_{1}, \ldots, \lambda_{r}$ are the different eigenvalues of $A$ then

$$
a(z)=\left(z-\lambda_{1}\right)^{m_{1}} \ldots\left(z-\lambda_{r}\right)^{m_{r}} .
$$

In that case $a_{j}(z)=\left(z-\lambda_{j}\right)$ and $F_{a_{j}}=\left(\lambda_{j}\right)$, and

$$
g_{j}(x)=\left[1, \lambda_{j}, \lambda_{j}^{2}, \ldots, \lambda_{j}^{n-1}\right] S\left[\begin{array}{c}
x_{0} \\
x_{1} \\
\vdots \\
x_{n-1}
\end{array}\right], j=1, \ldots, r .
$$

The factors $g_{j}(x)$ in (3.13) are related to the Popov-Belevitch-Hautus controllability test (see e.g. [11, p. 93]). It is known that - up multiplicative constants - the row vectors

$$
w_{j}^{\top}=\left[1, \lambda_{j}, \ldots, \lambda_{j}^{n-1}\right], j=1, \ldots, r,
$$

are the left eigenvectors of $F_{a}$. Then $S A S^{-1}=F_{a}$ implies that $v_{j}^{\top}=w_{j}^{\top} S$ are the left eigenvectors of $A$. Hence

$$
v_{j}^{\top} b=g_{j}(b), j=1, \ldots, r .
$$

Therefore we obtain the PBH criterion in the special case of cyclic matrices.

Corollary 3.3. Let $A$ be cyclic. The following statements are equivalent. (i) The pair $(A, b)$ is reachable. (ii) If

$$
v^{\top}(A-\lambda I)=0, v \in K^{n}, v \neq 0,
$$

then $v^{\top} b \neq 0$.

Proof. Because of (3.14) we can rewrite (ii) in the form

$$
g_{j}(b) \neq 0, j=1, \ldots, r .
$$


From (3.9) follows that (3.15) is equivalent to $\operatorname{det} R(A, b) \neq 0$.

We illustrate Theorem 3.2 with an example. Consider

$$
A=\left[\begin{array}{ccccc}
-6 & -38 & 6 & -4 & 281 \\
-11 & -131 & 10 & -5 & 928 \\
11 & -155 & -6 & -16 & 1191 \\
1 & -170 & 1 & -11 & 1253 \\
-1 & -21 & 1 & -1 & 151
\end{array}\right], \quad b=\left[\begin{array}{l}
3 \\
0 \\
4 \\
0 \\
0
\end{array}\right]
$$

Then

$$
R(A, b)=\left[\begin{array}{ccccc}
3 & 6 & 5 & 2 & 6 \\
0 & 7 & 0 & 0 & 8 \\
4 & 9 & 6 & 3 & 4 \\
0 & 7 & 1 & 0 & 5 \\
0 & 1 & 0 & 0 & 1
\end{array}\right], \operatorname{det} R(A, b)=1
$$

and the pair $(A, b)$ is reachable. Set $S=R(A, b)^{-1}$. Then

$$
S=\left[\begin{array}{ccccc}
3 & -16 & -2 & -3 & 133 \\
0 & -1 & 0 & 0 & 8 \\
0 & 2 & 0 & 1 & -21 \\
-4 & 19 & 3 & 2 & -150 \\
0 & 1 & 0 & 0 & -7
\end{array}\right]
$$

and $S A S^{-1}=F_{a}$. We have

$$
\begin{aligned}
& \operatorname{det}(z I-A)=a(z)=z^{5}+3 z^{4}-6 z^{3}-10 z^{2}-21 z-9= \\
&(z-1)^{3}(z+3)^{2}=\left(a_{1}(z)\right)^{3}\left(a_{2}(z)\right)^{2} .
\end{aligned}
$$

Hence $\operatorname{det} R(A, x)=g_{1}(x)^{3} g_{2}(x)^{2}$ with

$$
g_{1}(x)=[1,1,1,1,1] S x=-x_{0}+5 x_{1}+x_{2}-37 x_{4}
$$

and

$$
g_{2}(x)=[1,-3,9,-27,81] S x=111 x_{0}-427 x_{1}-83 x_{2}-48 x_{3}+3403 x_{4} .
$$

We conclude with some remarks which place our study into a larger context. Matrices of the form $\mathcal{K}_{r}(A, b)=\left[b, A b, \ldots, A^{r-1} b\right], 1 \leq r \leq n$, are known as Krylov matrices (see e.g. $\left[9\right.$, p. 646]). Thus $R(A, b)=\mathcal{K}_{n}(A, b)$. We refer to [8] for an investigation of numerical aspects of Krylov and reachability matrices. The concept of Faddeev reachability matrix was introduced in [5] and further elaborated in [10]. A "spectral factorization" of $R(A, b)$ is due to [7] (see also [13]). 


\section{REFERENCES}

[1] H. Bart and G. Ph. A. Thijsse. Simultaneous reduction to companion and triangular forms of sets of matrices. Linear Multilinear Algebra, 26:231-241, 1990.

[2] L. Brand. The companion matrix and its properties. Amer. Math. Monthly, 71:629-634, 1964.

[3] P. A. Fuhrmann. A Polynomial Approach to Linear Algebra. Springer, New York, 1996.

[4] I. Gohberg, P. Lancaster, and L. Rodman. Invariant Subspaces of Matrices with Applications. Wiley, New York, 1986.

[5] B. Hanzon and R. L. M. Peters. A Faddeev sequence method for solving Lyapunov and Sylvester equations. Linear Algebra Appl., 241:401-430, 1996.

[6] A. S. Householder. The Theory of Matrices in Numerical Analysis. Blaisdell, New York, 1964.

[7] I. C. F. Ipsen. Expressions and bounds for the GMRES residual. BIT, 40:524-535, 2000.

[8] A. Maćkiewicz, F. L. Almansa, and J. A. Inaudi. On Krylov matrices and controllability of n-dimensional linear time-invariant state equations. J. Structural Control, 3:99-109, 1996.

[9] C. D. Meyer. Matrix Analysis and Applied Linear Algebra. SIAM, Philadelphia, 2000.

[10] R. L. M. Peters and B. Hanzon. Symbolic computation of Fisher information matrices for parametrized state-space systems. Automatica, 35:1059-1071, 1999.

[11] E. D. Sontag. Mathematical Control Theory: Deterministic Systems. Springer, New York, 1990.

[12] J. H. Wilkinson. The Algebraic Eigenvalue Problem. Clarendon Press, Oxford, 1965.

[13] I. Zavorin, D. O'Leary, and H. Elman. Complete stagnation of GMRES. Linear Algebra Appl., 367:165-183, 2003. 